\title{
Stellungnahme zu E-Zigaretten
}

\author{
Schweizerische Gesellschaft \\ für Pneumologie (SGP) \\ Schweizerische Gesellschaft \\ für Pädiatrische Pneumologie \\ (SGPP)
}

Angesichts der Tatsache, dass die elektronische Zigarette (E-Zigarette) den Konsumenten und Konsumentinnen als Nikotinverabreichungssystem dienen kann und dabei die typischen Eigenschaften einer Tabakzigarette reproduziert, fordern die Schweizerische Gesellschaft für Pneumologie (SGP) und die Schweizerische Gesellschaft für Pädiatrische Pneumologie (SGPP) die entsprechenden Behörden und das Bundesamt für Gesundheit (BAG) auf, ihre rechtliche Zuständigkeit geltend $\mathrm{zu}$ machen und die E-Zigarette einer wirksamen Regulierung zu unterwerfen. Eine solche Regulierung muss die folgenden Punkte enthalten und sollte gewisse Zusatzaspekte mitberücksichtigen:

- Die SGP/SGPP empfehlen, am aktuellen Verbot des Verkaufs von nikotinhaltigen E-Zigaretten und Liquiden in der Schweiz festzuhalten, bis Klarheit über Nutzen und Risiken besteht. Die aktuelle Datenlage ist diesbezüglich ungenügend.
Gesundheit nicht gefährden dürfen, was bei E-Zigaretten nicht ausgeschlossen werden kann bzw. bei der Mehrheit der zurzeit erhältlichen Produkte erwartet werden muss (Tabakpräventionsmassnahme).

- Die bestehenden Werbe- und Sponsoring-Verbote/Einschränkungen für Tabakprodukte sollen in gleichem Masse auch für E-Zigaretten gelten. Die SGP/SGPP fordern ein gesamtschweizerisches Werbe- und Sponsoringverbot für alle Tabakprodukte und E-Zigaretten (Werbeverbot).

- E-Zigarettenpackungen sollten mit Warnhinweisen versehen sein, die in Grösse und Inhalt denen ähnlich sind, wie sie für die Packungen von brennbaren Tabakprodukten gelten. Über bekannte Risiken sollten die Konsumenten und Konsumentinnen in unmissverständlicher und direkter Sprache informiert werden. Wo keine Daten über Risiken vorliegen oder die Daten nicht schlüssig sind, sollten die Konsumenten

\section{Es wird empfohlen, am aktuellen Verbot des Verkaufs von nikotin- haltigen E-Zigaretten und Liquiden in der Schweiz festzuhalten.}

Korrespondenz:

Dr. med. Macé M. Schuurmans Co-Präsident Arbeitsgruppe Tabakprävention SGP Klinik für Pneumologie Universitätsspital Zürich Rämistrasse 100 CH-8091 Zürich

mace.schuurmans[at]usz.ch

PD Dr. med. Jürg Barben Sekretär SGPP Ostschweizer Kinderspital CH-9006 St. Gallen

juerg.barben[at]kispisg.ch
- Der Bund sollte E-Zigaretten mit oder ohne Nikotininhalt als Tabakprodukte behandeln. Die SGP/ SGPP fordern ein gesamtschweizerisches $\mathrm{Ab}$ gabeverbot von E-Zigaretten und Liquiden mit und ohne Nikotin an Personen unter 18 Jahren. Diese Forderung sollte auch gesamtschweizerisch für alle Tabakprodukte gelten (Kinder- und Jugendschutz). Gleichermassen sollte der Internetverkauf und Import von E-Zigaretten und Liquiden strikt reguliert werden.

- E-Zigaretten und Liquide, die Nikotin enthalten, sollten in gleicher Höhe besteuert werden wie traditionelle Zigaretten und andere Tabakprodukte (Tabaksteuer).

- Die öffentliche Nutzung von E-Zigaretten sollte denselben Beschränkungen unterliegen, wie sie für brennbare Tabakprodukte gelten: Der Gebrauch der E-Zigaretten (mit oder ohne Nikotin) in geschlossenen, öffentlich zugänglichen Räumen sollte, analog zur Regelung zum Schutz vor dem Passivrauch, verboten werden mit Bezug auf den Art. 14 LMG, wonach Gebrauchsgegenstände bei bestimmungsgemässem oder üblicherweise zu erwartendem Gebrauch die und Konsumentinnen über das Fehlen von verlässlichen Testdaten über die Unbedenklichkeit in Kenntnis gesetzt werden.

- Von E-Zigarettenherstellern gemachte explizite und implizite Aussagen über Gesundheit und Sicherheit sollten denselben Evidenzbeweisanforderungen unterliegen, wie sie gegenwärtig für ähnliche Aussagen über andere Produkte gelten.

- Der Inhalt von E-Zigarettenpatronen muss offengelegt, reguliert und kontrolliert werden. Der Nikotingehalt einer E-Zigarette darf den Nikotingehalt von 40 brennbaren Tabakzigaretten nicht überschreiten. Die Menge des abgegebenen Nikotins sollte bei allen Patronen einheitlich sein. Die Patronen müssen eine Kindersicherung aufweisen. Das BAG sollte von den E-Zigarettenherstellern fordern, dass sie dieselben Grundsätze der guten Herstellungspraxis anwenden, wie sie für andere regulierte Produkte gelten, wie z.B. Chargennummern und Sicherheitsverpackungen und Ähnliches.

- Angesichts der Tatsache, dass es sich bei Nikotin um eine süchtig machende Droge (Neurotoxin) 


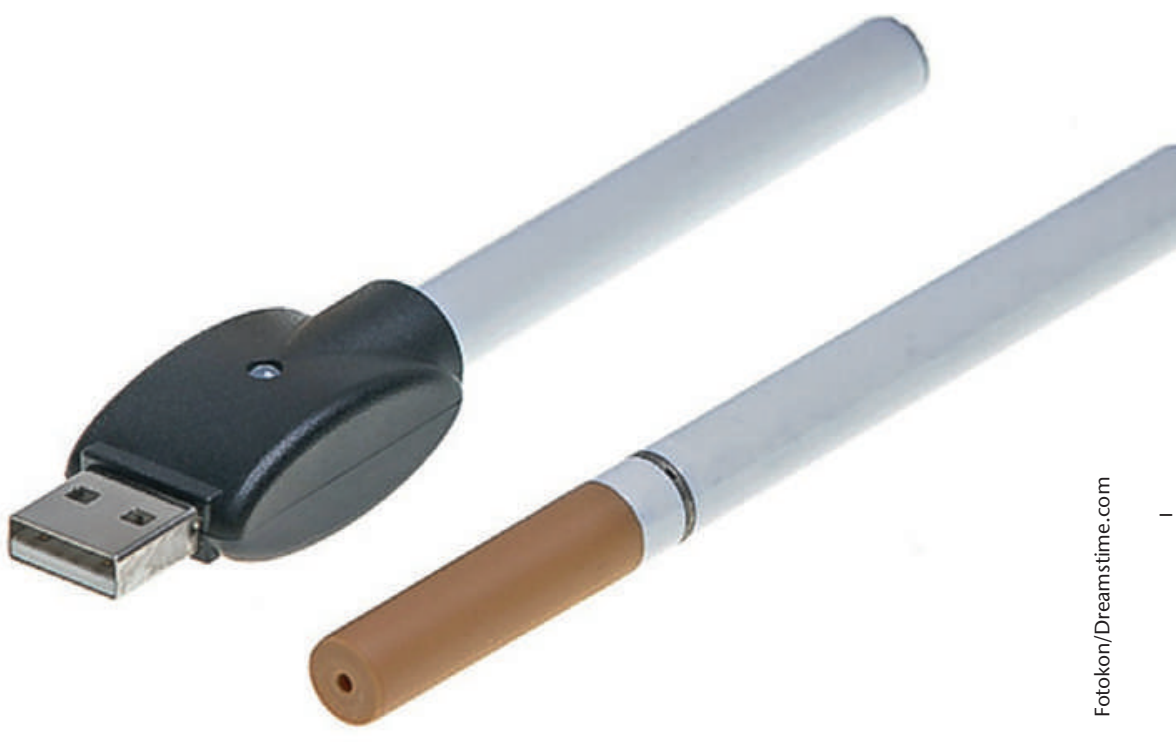

Der Inhalt von E-Zigarettenpatronen sollte offengelegt, reguliert und kontrolliert werden.

handelt und die Stärke der Suchterzeugung abhängig ist von den pharmakokinetischen Eigenschaften des Verabreichungssystems, sollten diese Systemeigenschaften geprüft, offengelegt und regelmässig kontrolliert werden, um so die Konstanz des Suchterzeugungspotentials des Produkts über die Zeit sicherzustellen.

- E-Zigaretten mit Nikotin werden teilweise als Unterstützung zur Raucherentwöhnung angepriesen, aber ihre tatsächliche Wirksamkeit als Mittel zur Raucherentwöhnung wird auch unter

\section{Die WHO rät zum jetzigen Zeitpunkt von E-Zigaretten als Mittel zur Raucherentwöhnung ab.}

den Experten noch kontrovers diskutiert. Deshalb rät auch die WHO zum jetzigen Zeitpunkt von E-Zigaretten als Mittel zur Raucherentwöhnung ab. Das Ziel der Behandlung einer Tabakabhängigkeit ist die anhaltende und nachgewiesene Abstinenz vom Nikotinkonsum. Ein erfolgreicher Rauchstopp ist somit eine Nikotin- freiheit. Diese Definition soll bei der Beurteilung von Entwöhnungsmassnahmen zur Tabakabhängigkeit angewendet werden.

- Die SGP/SGPP fordern, dass alle Forscherund Kliniker sowie wissenschaftliche Gesellschaften und Publikationsorgane, die von E-Zigarettenherstellern finanziell unterstützt werden, diese Beziehungen und die damit einhergehenden Möglichkeiten von Interessenkonflikten in derselben Weise offenlegen, wie dies für Beziehungen zur Tabakindustrie verlangt wird.

- Die SGP/SGPP haben grosse Bedenken bezüglich der Tatsache, dass die Tabakindustrie den Markt der E-Zigaretten beherrscht. Die Ähnlichkeit des Produktes mit der konventionellen Zigarette und der Vertrieb über dieselbe Industrie stellen einen fundamentalen Konflikt dar.

\section{Literatur}

- Eidgenössische Kommission für Tabakprävention. Stellungnahme zu E-Zigaretten. August 2012.

- American Thoracic Society: ATS Policy eCigarettes, http://news.thoracic.org/november-2013/ the-advocate.php

- International Union Against Tuberculosis and Lung Disease. Position statement on electronic cigarettes or electronic nicotine delivery systems (Int J Tuberc Lung Dis. 2014;18(1):5-7.

- Britton J. Electronic cigarettes. Thorax published online August 12, 2013 doi: 10.1136/thoraxjnl-2013-203973.

- Bullen C et al. Electronic cigarettes for smoking cessation: a randomised controlled trial. Lancet. Published Online September 7, 2013 http://dx.doi. org/10.1016/ S0140-6736(13)61842-

- Fairchaild AL et al. The Renormalization of Smoking? E-Cigarettes and the Tobacco «Endgame». N Engl J Med. December 18; 2013.

- De Andrade M et al. Promotion of electronic cigarettes: tobacco marketing reinvented? BMJ. 2013;347:f7473 doi: 10.1136/bmj.f7473

- Drummond MB et al. Electronic cigarettes. Potential harms and benefits. Ann Am Thorax Soc. 2014;11: 236-42.

- Elektrische Zigaretten - ein Überblick. Deutsches Krebsforschungsinstitut. Rote Reihe, Tabakprävention und Tabakkontrolle, Band 19. Deutsches Krebsforschungszentrum. Heidelberg; 2013. 